\title{
ISOTROPIC GEOMETRY OF GRAPH SURFACES ASSOCIATED WITH PRODUCT PRODUCTION FUNCTIONS IN ECONOMICS
}

\author{
MUHITTIN EVREN AYDIN AND MAHMUT ERGUT
}

\begin{abstract}
A production function is a mathematical formalization in economics which denotes the relations between the output generated by a firm, an industry or an economy and the inputs that have been used in obtaining it. In this paper, we study the product production functions of 2 variables in terms of the geometry of their associated graph surfaces in the isotropic 3-space $\mathbb{\natural}^{3}$. In particular, we derive several classification results for the graph surfaces of product production functions in $\mathbb{1}^{3}$ with constant curvature.
\end{abstract}

\section{Introduction}

In economics, a production function is a non-constant positive function that specifies the output of a firm, an industry, or an entire economy for all combinations of inputs. Explicitly, it is a map of class $C^{\infty}$ which has non-vanishing first derivatives defined by

$$
\left\{\begin{array}{l}
h: \mathbb{R}_{+}^{n} \longrightarrow \mathbb{R}_{+},\left(x_{1}, x_{2}, \ldots, x_{n}\right) \longmapsto h\left(x_{1}, x_{2}, \ldots, x_{n}\right), \\
\mathbb{R}_{+}^{n}=\left\{\left(x_{1}, x_{2}, \ldots, x_{n}\right): x_{i}>0, i=1, \ldots, n\right\} .
\end{array}\right.
$$

Here $h$ denotes the quantity of output, $n$ is the number of inputs and the variables are the inputs (such as capital, labor, raw materials etc.). Some interesting examples of production functions can be found in [18].

In order for the production functions to model as well economic reality, they are required to get some proporties (see e.g. [5, 22]). The most important of these proporties is the production function $f$ to be homogeneous, i.e. there exist a real number $p$ such that

$$
h\left(\lambda x_{1}, \lambda x_{2}, \ldots, \lambda x_{n}\right)=\lambda^{p} h\left(x_{1}, x_{2}, \ldots, x_{n}\right), \lambda \in \mathbb{R}_{+} .
$$

(1.2) implies that when the inputs are multiplied by same factor, the output is multiplied by some power of the factor.

Received March 1, 2016, accepted May 25, 2016.

2010 Mathematics Subject Classification. 91B38, 53A35, 53A40, 53B25.

Key words and phrases. Production function, return to scale, relative curvature, isotropic mean curvature, isotropic space.

Corresponding author: Muhittin Evren Aydin. 
If $p<1$ (resp. $p>1$ ) in (1.2), then the production function is said to have decreasing (resp. increasing) return to scale. If $p=1$, then it is said to have constant return to scale.

The presence of increasing returns means that a one percent increase in the usage levels of all inputs would result in a greater than one percent increase in output; the presence of decreasing returns means that it would result in a less than one percent increase in output. Constant returns to scale is the in-between case (cf. [8]).

A. D. Vilcu and G. E. Vilcu [26] completely classified the homogeneous production functions with constant proportional marginal rate of substitution. Further, the homogeneous production functions have been investigated via their associated graph hypersurfaces in [6][11], [29].

The most famous one among homogeneous production functions is Cobb-Douglas production function, introduced in 1928 by C. W. Cobb and P. H. Douglas [12]. In original form, it is given as

$$
Y=b L^{k} C^{1-k},
$$

where $b$ presents the total factor productivity, $Y$ the total production, $L$ the labor input and $C$ the capital input.

The generalized Cobb-Douglas production function of $n$ variables is defined by

$$
h(\mathbf{x})=A x_{1}^{\alpha_{1}} x_{2}^{\alpha_{2}} \cdots x_{n}^{\alpha_{n}}, \mathbf{x}=\left(x_{1}, x_{2}, \ldots, x_{n}\right) \in \mathbb{R}_{+}^{n}
$$

where $A, \alpha_{1}, \alpha_{2}, \ldots, \alpha_{n}>0$. We note that $h$ has constant return to scale if and only if $\sum_{i=1}^{n} \alpha_{i}=$ 1.

X. Wang, Y. Fu [28] and A.D. Vilcu, G.E. Vilcu [24, 25] classified the graph hypersurfaces of the generalized Cobb-Douglas production functions with zero Gauss-Kronocker and mean curvature.

On the other hand, there are some non-homogeneous production functions, including the famous Spillman-Mitscherlich and transcendental production functions respectively defined by

$$
\left\{\begin{array}{l}
h(\mathbf{x})=A\left[1-\exp \left(a_{1} x_{1}\right)\right] \cdot\left[1-\exp \left(a_{2} x_{2}\right)\right] \cdots\left[1-\exp \left(a_{n} x_{n}\right)\right] \\
\mathbf{x}=\left(x_{1}, x_{2}, \ldots, x_{n}\right) \in \mathbb{R}_{+}^{n}, A>0, a_{i}<0, i=1, \ldots, n
\end{array}\right.
$$

and

$$
\left\{\begin{array}{l}
h(\mathbf{x})=A x_{1}^{a_{1}} \exp \left(b_{1} x_{1}\right) \cdot x_{2}^{a_{2}} \exp \left(b_{2} x_{2}\right) \cdots x_{n}^{a_{n}} \exp \left(b_{n} x_{n}\right), \\
\mathbf{x}=\left(x_{1}, x_{2}, \ldots, x_{n}\right) \in \mathbb{R}_{+}^{n}, A>0, a_{i}, b_{i} \in \mathbb{R}, a_{i}^{2}+b_{i}^{2} \neq 0, i=1, \ldots, n .
\end{array}\right.
$$

Such production functions, including the generalized Cobb-Douglas production function, belong to a more general class of production functions given by

$$
h(\mathbf{x})=\prod_{j=1}^{n} g_{j}\left(x_{j}\right), \mathbf{x}=\left(x_{1}, x_{2}, \ldots, x_{n}\right) \in \mathbb{R}_{+}^{n},
$$


where $g_{j}$ is a continuous positive real function with nonzero first derivatives, $j=1, \ldots, n$. H. Alodan et al. [1] called these production functions product production functions. A production function is said to be quasi-product, if it is of the form

$$
h(\mathbf{x})=F\left(\prod_{j=1}^{n} g_{j}\left(x_{j}\right)\right), \mathbf{x}=\left(x_{1}, x_{2}, \ldots, x_{n}\right) \in \mathbb{R}_{+}^{n},
$$

where $F, g_{j}$ are continuous positive real functions with nonzero first derivatives, $j=1, \ldots, n$. A lot of classifications of the quasi-product and quasi-sum (see [16]) production functions can be found in [1]-[4], [14, 27] in terms of the geometry of their graph hypersurfaces.

B.-Y. Chen, et al. [7, 13] investigated the graph hypersurfaces of the production models via the isotropic geometry. In the present paper, we classify the graph surfaces of product production functions of 2 variables with constant curvature in the isotropic 3 -space $\rrbracket^{3}$.

\section{Basics on isotropic spaces}

For later use, we provide a brief review of isotropic geometry from [7], [19]-[21].

The isotropic 3-space $\rrbracket^{3}$ is a Cayley-Klein space defined from a 3-dimensional projective space $P\left(\mathbb{R}^{3}\right)$ with the absolute figure which is an ordered triple $\left(\omega, f_{1}, f_{2}\right)$, where $\omega$ is a plane in $P\left(\mathbb{R}^{3}\right)$ and $f_{1}, f_{2}$ are two complex-conjugate straight lines in $\omega$. The homogeneous coordinates in $P\left(\mathbb{R}^{3}\right)$ are introduced in such a way that the absolute plane $\omega$ is given by $X_{0}=0$ and the absolute lines $f_{1}, f_{2}$ by $X_{0}=X_{1}+i X_{2}=0, X_{0}=X_{1}-i X_{2}=0$. The intersection point $F(0: 0: 0: 1)$ of these two lines is called the absolute point. The group of motions $G_{6}$ of $\square^{3}$ is a six-parameter group given in the affine coordinates $x_{1}=\frac{X_{1}}{X_{0}}, x_{2}=\frac{X_{2}}{X_{0}}, x_{3}=\frac{X_{3}}{X_{0}}$ by

$$
\left(x_{1}, x_{2}, x_{3}\right) \longmapsto\left(x_{1}^{\prime}, x_{2}^{\prime}, x_{3}^{\prime}\right):\left\{\begin{array}{l}
x_{1}^{\prime}=a+x_{1} \cos \phi-x_{2} \sin \phi, \\
x_{2}^{\prime}=b+x_{1} \sin \phi+x_{2} \cos \phi, \\
x_{3}^{\prime}=c+d x_{1}+e x_{2}+x_{3},
\end{array}\right.
$$

where $a, b, c, d, e, \phi \in \mathbb{R}$. Such affine transformations are called isotropic congruence transformations or $i$-motions. It is easily seen from (2.1) that i-motions are indeed composed by an Euclidean motion in the $x_{1} x_{2}$-plane (i.e. translation and rotation) and an affine shear transformation in $x_{3}$-direction.

In general, the following terminology is used for the isotropic spaces. Consider the points $\mathbf{x}=\left(x_{1}, x_{2}, x_{3}\right)$ and $\mathbf{y}=\left(y_{1}, y_{2}, y_{3}\right)$. The projection in $\mathbf{z}$-direction onto $\mathbb{R}^{2},\left(x_{1}, x_{2}, x_{3}\right) \longmapsto\left(x_{1}, x_{2}, 0\right)$, is called the top view. In the sequel, many of metric properties in isotropic geometry (invariants under $G_{6}$ ) are Euclidean invariants in the top view such as the isotropic distance, socalled i-distance. I-distance of two points $\mathbf{x}$ and $\mathbf{y}$ is defined as the Euclidean distance of their 
top views, i.e.,

$$
\|\mathbf{x}-\mathbf{y}\|_{i}=\sqrt{\sum_{j=1}^{2}\left(y_{j}-x_{j}\right)^{2}}
$$

The i-metric is degenerate along the lines in $\mathbf{z}$-direction, and such lines are called isotropic lines. The plane containing an isotropic line is called an isotropic plane. Therefore, an isotropic 3 -space $\square^{3}$ is the product of the Euclidean 2-space $\mathbb{R}^{2}$ and an isotropic line with a degenerate parabolic distance metric.

Let $M^{2}$ be a regular surface immersed in $\rrbracket^{3}$ which has no isotropic tangent planes. Such a surface $M^{2}$ is said to be admissible and can be parametrized by

$$
r: D \subseteq \mathbb{R}^{2} \longrightarrow \mathbb{1}^{3}:\left(u_{1}, u_{2}\right) \longmapsto\left(r_{1}\left(u_{1}, u_{2}\right), r_{2}\left(u_{1}, u_{2}\right), r_{3}\left(u_{1}, u_{2}\right)\right),
$$

where $r_{1}, r_{2}$ and $r_{3}$ are smooth real-valued functions on a domain $D \subseteq \mathbb{R}^{2}$. Denote $g$ the metric on $M^{2}$ induced from $\rrbracket^{3}$. The components of the first fundamental form of $M^{2}$ can be calculated via the induced metric $g$ as follows

$$
g_{i j}=g\left(r_{u_{i}}, r_{u_{j}}\right), r_{u_{i}}=\frac{\partial r}{\partial u_{i}}, i, j \in\{1,2\} .
$$

The unit normal vector field of $M^{2}$ is completely isotropic, i.e. $(0,0,1)$. Also, the components of the second fundamental form are (for details, see [20], p. 150-155)

$$
t_{i j}=\frac{\operatorname{det}\left(r_{u_{i} u_{j}}, r_{u_{1}}, r_{u_{2}}\right)}{\sqrt{\operatorname{det}\left(g_{i j}\right)}}, r_{u_{i} u_{j}}=\frac{\partial^{2} r}{\partial u_{i} \partial u_{j}}, i, j \in\{1,2\}
$$

Thus the relative curvature (so-called the isotropic curvature or isotropic Gaussian curvature) and the isotropic mean curvature are respectively defined by

$$
K=\frac{\operatorname{det}\left(t_{i j}\right)}{\operatorname{det}\left(g_{i j}\right)}, H=\frac{g_{11} t_{22}-2 g_{12} t_{12}+g_{22} t_{11}}{2 \operatorname{det}\left(g_{i j}\right)} .
$$

A surface is called isotropic minimal (resp. isotropic flat) if its isotropic mean curvature (resp. relative curvature) vanishes.

In particular, if $M^{2}$ is a Monge surface in $\rrbracket^{3}$ of the form

$$
\left(u_{1}, u_{2}\right) \longmapsto\left(u_{1}, u_{2}, h\left(u_{1}, u_{2}\right)\right),
$$

then the metric on $M^{2}$ induced from $\mathbb{1}^{3}$ is given by $g_{*}=d u_{1}^{2}+d u_{2}^{2}$. This implies that $M^{2}$ is always a flat space with respect to the induced metric $g_{*}$. Thus its Laplacian is given by

$$
\triangle=\sum_{j=1}^{2} \frac{\partial^{2}}{\partial u_{j}^{2}}
$$


Also, the matrix of second fundamental form of $M^{2}$ becomes the Hessian matrix of $h$ (i.e. the square matrix $\left(h_{u_{i} u_{j}}\right)$ of second-order partial derivatives of the function $\left.h\right)$. Thereby, the formulas in (2.5) reduce to

$$
K=\operatorname{det}\left(h_{u_{i} u_{j}}\right)=h_{u_{1} u_{1}} h_{u_{2} u_{2}}-\left(h_{u_{1} u_{2}}\right)^{2}, 2 H=\Delta h=h_{u_{1} u_{1}}+h_{u_{2} u_{2}} .
$$

\section{Product production functions}

Let us consider the product production function of 2 variables given by

$$
h: \mathbb{R}_{+}^{2} \longrightarrow \mathbb{R}_{+},(x, y) \longmapsto h(x, y)=f(x) g(y),
$$

where $f, g$ are continuous positive real functions with $f^{\prime}(x)=\frac{d f}{d x} \neq 0$ and $g^{\prime}(y)=\frac{d g}{d y} \neq 0$. The graph surface $M^{2}$ corresponding to $h$ is of the form

$$
r(x, y)=(x, y, h(x, y)=f(x) g(y))
$$

which we call product production surface.

We remark that the surfaces of the form (3.1) are known as factorable surfaces or homothetical surfaces in ambient spaces and have been studied in [15, 17, 23, 30].

The following result provides a complete classification of the product production surfaces in $\rrbracket^{3}$ with constant relative curvature.

Theorem 3.1. Let $M^{2}$ be a surface given by (3.1) in $\rrbracket^{3}$ with constant relative curvature $K_{0}$.

(A) If $K_{0}=0$, then one of the following occurs:

(A.1) $h(x, y)=c_{1} f(x)$ or $h(x, y)=c_{2} g(y)$ for nonzero constants $c_{1}, c_{2}$.

(A.2) $h$ is a transcendental production function of 2 variables given by

$$
h(x, y)=A \exp \left(c_{1} x+c_{2} y\right) \text {, }
$$

where $A, c_{1}, c_{2}$ are nonzero constants.

(A.3) Up to translations of $x$ and $y, h$ is a Cobb-Douglas production function of 2 variables with constant return to scale.

(B) If $K_{0} \neq 0$, then it is negative $\left(K_{0}<0\right)$ and, up to translations of $x$ and $y, h$ is a Cobb-Douglas production function of 2 variables given by

$$
h(x, y)=\sqrt{-K_{0}} x y .
$$


Proof. Assume that $M^{2}$ has constant relative curvature $K_{0}$ in $\rrbracket^{3}$. Then, it follows from (2.6) that

$$
\left(f^{\prime \prime} g^{\prime \prime}\right) f g-\left(f^{\prime} g^{\prime}\right)^{2}=K_{0}
$$

where $f^{\prime}=\frac{d f}{d x}$ and $g^{\prime}=\frac{d g}{d y}$, etc. We divide the proof into two cases:

Case (i). $K_{0}=0$. Then, from (3.2), both situations, $f$ or $g$ constants, are solutions for (3.2). This implies the statement (A.1) of the theorem. Now, let us assume that $f$ and $g$ are nonconstant functions. Hence, it follows from (3.2) that $f$ and $g$ cannot be linear functions. Thus the equation (3.2) can be rewritten as

$$
\frac{f^{\prime \prime} f}{\left(f^{\prime}\right)^{2}}-\frac{\left(g^{\prime}\right)^{2}}{g^{\prime \prime} g}=0
$$

which yields

$$
\frac{f f^{\prime \prime}}{\left(f^{\prime}\right)^{2}}=\lambda=\frac{\left(g^{\prime}\right)^{2}}{g^{\prime \prime} g}
$$

for a nonzero constant $\lambda$. In order to solve (3.3) we have to distinguish two situations.

Case (i.1). $\lambda=1$. Then after solving (3.2) we get

$$
f(x)=c_{1} \exp \left(c_{2} x\right) \text { and } g(y)=c_{3} \exp \left(c_{4} y\right),
$$

where $c_{i}$ are nonzero constants, $1 \leq i \leq 4$, which gives the the statement (A.2) of the theorem.

Case (i.2). $\lambda \neq 1$. Solving (3.3) yields

$$
f(x)=\left[(1-\lambda)\left(c_{1} x+d_{1}\right)\right]^{\frac{1}{1-\lambda}} \text { and } g(y)=\left[\left(\frac{\lambda-1}{\lambda}\right)\left(c_{2} y+d_{2}\right)\right]^{\frac{\lambda}{\lambda-1}}
$$

for nonzero constants $c_{1}, c_{2}$ and some constants $d_{1}, d_{2}$. Up to suitable translations of $x, y$, we obtain

$$
h(x, y)=f(x) g(y)=A x^{\frac{1}{1-\lambda}} y^{-\frac{\lambda}{1-\lambda}}
$$

for $A=\left[c_{1}(1-\lambda)\right]^{\frac{1}{1-\lambda}}\left[c_{2}\left(\frac{\lambda-1}{\lambda}\right)\right]^{\frac{\lambda}{\lambda-1}}$. This proves the statement (A.3) of the theorem.

Case (ii). $K_{0} \neq 0$. Suppose that $f$ and $g$ are non-linear functions. Hence, we can rewrite (3.2) as

$$
\frac{f f^{\prime \prime}}{\left(f^{\prime}\right)^{2}}-\frac{\left(g^{\prime}\right)^{2}}{g g^{\prime \prime}}=\frac{K_{0}}{\left(f^{\prime}\right)^{2} g g^{\prime \prime}}
$$

Differentiating of (3.4) with respect to $y$ gives

$$
-\left(\frac{\left(g^{\prime}\right)^{2}}{g g^{\prime \prime}}\right)^{\prime}=\frac{K_{0}}{\left(f^{\prime}\right)^{2}}\left(\frac{1}{g g^{\prime \prime}}\right)^{\prime}
$$


From (3.5), if

$$
\left(\frac{1}{g g^{\prime \prime}}\right)^{\prime}=0
$$

i.e. $g g^{\prime \prime}$ is a nonzero constant $c$ in (3.5), then we get $\frac{1}{c}\left(\left(g^{\prime}\right)^{2}\right)^{\prime}=0$, which is not possible because $g$ is non-linear function. In (3.5) if

$$
\left(\frac{\left(g^{\prime}\right)^{2}}{g g^{\prime \prime}}\right)^{\prime}=0,
$$

then (3.5) reduces to

$$
\frac{K_{0}}{\left(f^{\prime}\right)^{2}}\left(\frac{1}{g g^{\prime \prime}}\right)^{\prime}=0,
$$

which yields $g g^{\prime \prime}=d, d \neq 0$. Considering this in (3.6) implies $\frac{2}{d} g^{\prime} g^{\prime \prime}=0$ and it is a contradiction. Thereby we can rewrite (3.5) as

$$
-\frac{\left(\frac{\left(g^{\prime}\right)^{2}}{g g^{\prime \prime}}\right)^{\prime}}{\left(\frac{1}{g g^{\prime \prime}}\right)^{\prime}}=\frac{K_{0}}{\left(f^{\prime}\right)^{2}} .
$$

Since $f$ is a non-linear function, the right-side of (3.7) is a function of $x$. However the left-side of (3.7) is either a constant or a function of $y$. Both cases are not possible.

Now let either $f$ or $g$ be a linear function. Without loss of generality, we may assume that $f$ is a linear function, i.e. $f(x)=c_{1} x+d_{1}, c_{1} \neq 0, d_{1} \in \mathbb{R}$. Then we get from (3.2)

$$
g^{\prime}=\frac{\sqrt{-K_{0}}}{c_{1}}, K_{0}<0 .
$$

This implies that $g$ is also a linear function, i.e. $g(y)=\frac{\sqrt{-K_{0}}}{c_{1}} y+d_{2}, d_{2} \in \mathbb{R}$. Thus, up to suitable translations of $x$ and $y$, we derive

$$
h(x, y)=\sqrt{-K_{0}} x y \text {. }
$$

This gives of the statement (B) of the theorem.

Therefore, the proof is completed.

Next classifies the product production surfaces of constant isotropic mean curvature in $\square^{3}$.

Theorem 3.2. Let $M^{2}$ be a surface given by (3.1) in $\square^{3}$ with constant isotropic mean curvature $H_{0}$. 
(A) If $\mathrm{H}_{0}$ is a nonzero constant, then

$$
h(x, y)=\frac{H_{0}}{g_{0}} x^{2}+d_{1} x+d_{2},\left(\text { or } \frac{H_{0}}{f_{0}} y^{2}+d_{1} y+d_{2}\right)
$$

for $f_{0}, g_{0} \in \mathbb{R}-\{0\}, d_{1}, d_{2} \in \mathbb{R}$.

(B) If $M^{2}$ is isotropic minimal, i.e. $H_{0}=0$, then either

(B.1) up translations of $x$ and $y, h$ is a Cobb-Douglas production function of 2 variables given by $h(x, y)=A x y, A>0$, or

(B.2) $h$ is given by one of the following

$$
h(x, y)=\left(c_{1} e^{\sqrt{\lambda} x}+c_{2} e^{-\sqrt{\lambda} x}\right)\left(c_{3} \cos (\sqrt{\lambda} y)+c_{4} \sin (\sqrt{\lambda} y)\right)
$$

and

$$
h(x, y)=\left(c_{5} \cos (\sqrt{-\lambda} x)+c_{6} \sin (\sqrt{-\lambda} x)\right)\left(c_{7} e^{\sqrt{-\lambda} y}+c_{8} e^{-\sqrt{-\lambda} y}\right)
$$

for nonzero constants $\lambda, c_{1}, \ldots, c_{8}$.

Proof. Assume that $M^{2}$ has constant isotropic mean curvature $H_{0}$. Then, by (2.6), we get

$$
2 H_{0}=f^{\prime \prime} g+f g^{\prime \prime}
$$

It follows from (3.8) that when $g$ is a nonzero constant $g_{0}$ we have

$$
f(x)=\frac{H_{0}}{g_{0}} x^{2}+d_{1} x+d_{2}, d_{1}, d_{2} \in \mathbb{R},
$$

and analogously if $f$ is a nonzero constant $f_{0}$, we deduce

$$
g(y)=\frac{H_{0}}{f_{0}} y^{2}+d_{3} y+d_{4}, d_{3}, d_{4} \in \mathbb{R},
$$

which proves the statement (A) of the theorem.

Now suppose that $f, g$ are non-constant functions. Then (3.8) can be rewritten as

$$
\frac{f^{\prime \prime}}{f}+\frac{g^{\prime \prime}}{g}=\frac{2 H_{0}}{f g} .
$$

After taking the partial derivative of (3.9) with respect to $x$, we deduce

$$
\left(\frac{f^{\prime \prime}}{f}\right)^{\prime} \frac{f^{2}}{f^{\prime}}=-2 H_{0} \frac{1}{g}
$$

The left-side of (3.10) is etiher a constant or a function of $x$ while the other side is a function of $y$. This case is only possible when $H_{0}=0$ and

$$
\left(\frac{f^{\prime \prime}}{f}\right)^{\prime}=0
$$


Similarly, taking the partial derivative of (3.9) with respect to $y$, we find $H_{0}=0$ and

$$
\left(\frac{g^{\prime \prime}}{g}\right)^{\prime}=0 \text {. }
$$

This means that $M^{2}$ is isotropic minimal, i.e. $H_{0}=0$. From (3.9), (3.11) and (3.12), we conclude

$$
\frac{f^{\prime \prime}}{f}=\lambda=-\frac{g^{\prime \prime}}{g}
$$

for some constant $\lambda$. If $\lambda=0$ in (3.13), then $f$ and $g$ become linear functions. Thus, up translations of $x$ and $y$, we get $h(x, y)=A x y$, which gives the proof of the statement (B.1).

Now let us assume that $\lambda \neq 0$, i.e. $f$ and $g$ are non-linear functions. Then the equations in (3.13) can be rewritten as

$$
f^{\prime \prime}-\lambda f=0
$$

and

$$
g^{\prime \prime}+\lambda g=0
$$

If $\lambda>0$, by solving (3.14) and (3.15), we derive

$$
f(x)=c_{1} e^{\sqrt{\lambda} x}+c_{2} e^{-\sqrt{\lambda} x}
$$

and

$$
g(y)=c_{3} \cos (\sqrt{\lambda} y)+c_{4} \sin (\sqrt{\lambda} y)
$$

for nonzero constants $c_{1}, \ldots, c_{4}$. Otherwise (i.e. $\lambda<0$ ) we obtain

$$
f(x)=c_{5} \cos (\sqrt{-\lambda} y)+c_{6} \sin (\sqrt{-\lambda} y)
$$

and

$$
g(y)=c_{7} e^{\sqrt{-\lambda} y}+c_{8} e^{-\sqrt{-\lambda} y}
$$

for nonzero constants $c_{5}, \ldots, c_{8}$. This gives the proof.

Remark 3.3. For the product production function given by $h(x, y)=f(x) g(y)$, we have that $f$ and $g$ are nonconstant functions. Hence, while the statement (A.1) of Theorem 3.1 and the statement (A) of Theorem 3.2 are correct in mathematical perspective, in reality such product production functions do not exist.

Now let us consider Spillman-Mitscherlich and transcendental production functions of 2 variables respectively given by

$$
\left\{\begin{array}{l}
h(x, y)=A[1-\exp (a x)] \cdot[1-\exp (b y)], \\
(x, y) \in \mathbb{R}_{+}^{2}, A>0, a, b<0
\end{array}\right.
$$


and

$$
\left\{\begin{array}{l}
h(x, y)=A x^{a_{1}} \exp \left(b_{1} x_{1}\right) \cdot x_{2}^{a_{2}} \exp \left(b_{2} x_{2}\right), \\
(x, y) \in \mathbb{R}_{+}^{2}, A>0, a_{i}, b_{i} \in \mathbb{R}, a_{i}^{2}+b_{i}^{2} \neq 0, i=1,2 .
\end{array}\right.
$$

From Theorem 3.1 and Theorem 3.2, we obtain the following results for the surfaces corresponding to these production functions.

Corollary 3.4. Let $h$ be a Spillman-Mitscherlich production function of 2 variables given by (3.16). Then the corresponding graph surface $M^{2}$ of h has neither constant relative nor constant isotropic curvature in $\rrbracket^{3}$.

Corollary 3.5. Let $h$ be a transcendental production function of 2 variables given by (3.17) and $M^{2}$ its associated graph surface in $\square^{3}$. Then:

(A) $M^{2}$ has constant relative curvature $K_{0}$ in $\square^{3}$ if and only if $K_{0}=0$ and one of the following occurs:

(A.1) $a_{1}=a_{2}=0$ and $b_{1} \neq 0 \neq b_{2}$, or

(A.2) $a_{1}+a_{2}=1, a_{1} \neq 0 \neq a_{2}$ and $b_{1}=b_{2}=0$.

(B) $M^{2}$ has constant isotropic curvature $H_{0}$ in $\square^{3}$ if and only if $H_{0}=0$ and $a_{1}=a_{2}=1, b_{1}=b_{2}=$ 0.

\section{References}

[1] H. Alodan, B.-Y. Chen, S. Deshmukh and G. E. Vilcu, On some geometric properties of quasi-product production models, arXiv:1512.05190v1 [math.DG], 2015.

[2] M. E. Aydin and M. Ergut, Hessian determinants of composite functions with applications for production functions in economics, Kragujevac J. Math., 38(2) (2014), 259-268.

[3] M. E. Aydin and A. Mihai, Classifications of quasi-sum production functions with Allen determinants, Filomat, 29 (2015), 1351-1359.

[4] M. E. Aydin and M. Ergut, Composite functions with Allen determinants and their applications to production models in economics, Tamkang J. Math., 45 (2014), 427-435.

[5] R. G. Chambers, Applied Production Analysis, Cambridge University Press, 1998.

[6] B.-Y. Chen and G. E. Vîlcu, Geometric classifications of homogeneous production functions, Appl. Math. Comput., 225 (2013), 345-351.

[7] B.-Y. Chen, S. Decu and L. Verstraelen, Notes on isotropic geometry of production models, Kragujevac J. Math., 38 (2014), 23-33.

[8] B.-Y. Chen, On some geometric properties of h-homogeneous production function in microeconomics, Kragujevac J. Math., 35 (2011), 343-357.

[9] B.-Y. Chen, On some geometric properties of quasi-sum production models, J. Math. Anal. Appl., 392 (2012), 192-199.

[10] B.-Y. Chen, Classification of h-homogeneous production functions with constant elasticity of substitution, Tamkang J. Math., 43 (2012), 321-328.

[11] B.-Y. Chen, Solutions to homogeneous Monge-Ampere equations of homothetic functions and their applications to production models in ecenomics, J. Math. Anal. Appl., 411 (2014), 223-229. 
[12] C. W. Cobb and P. H. Douglas, A theory of production, Amer. Econom. Rev., 18(1928), 139-165.

[13] S. Decu and L. Verstraelen, A note on the isotropical geometry of production surfaces, Kragujevac J. Math., 37 (2013), 217-220.

[14] Y. Fu, Geometric characterizations of quasi-product production models in economics, Filomat, (2016), to be published.

[15] R. Lopez and M. Moruz, Translation and homothetical surfaces in Euclidean space with constant curvature, J. Korean Math. Soc., 52 (2015), 523-535.

[16] L. Losonczi, Production functions having the CES property, Acta Math. Acad. Paedagog. Nyhái. (N.S.) 26 (2010), 113-125.

[17] H. Meng and H. Liu, Factorable surfaces in Minkowski space, Bull. Korean Math. Soc., 46 (2009), $155-169$.

[18] S. K. Mishra, A brief history of production functions, IUP J. Manage. Econom., 8(4) (2010), 6-34.

[19] H. Pottmann, P. Grohs and N. J. Mitra, Laguerre minimal surfaces, isotropic geometry and linear elasticity, Adv. Comput. Math., 31 (2009), 391-419.

[20] H. Sachs, Isotrope Geometrie des Raumes, Vieweg, 1990.

[21] Z. M. Sipus, Translation surfaces of constant curvatures in a simply isotropic space, Period. Math. Hung., 68 (2014), 160-175.

[22] A. Thompson, Economics of the Firm, Theory and Practice, 3rd edition, Prentice Hall, 1981.

[23] I. Van de Woestyne, Minimal homothetical hypersurfaces of a semi-Euclidean space, Results Math., 27 (1995), 333-342.

[24] G. E. Vîlcu, A geometric perspective on the generalized Cobb-Douglas production functions, Appl. Math. Lett., 24 (2011), 777-783.

[25] A. D. Vilcu and G. E. Vilcu, On some geometric properties of the generalized CES production functions, Appl. Math. Comput., 218 (2011), 124-129.

[26] A. D. Vilcu and G. E. Vilcu, On homogeneous production functions with proportional marginal rate of substitution, Math. Probl. Eng. 2013 (2013), Article ID 732643, 5 pages.

[27] A. D. Vilcu and G. E. Vilcu, Some characterizations of the quasi-sum production models with proportional marginal rate of substitution, C. R. Math. Acad. Sci. Paris, 353 (2015), 1129-1133.

[28] X. Wang and Y. Fu, Some characterizations of the Cobb-Douglas and CES production functions in microeconomics, Abstr. Appl. Anal. 2013 (2013), Article ID 761832, 6 pages.

[29] X. Wang, A geometric characterization of homogeneous production models in economics, Filomat, (2016), to be published.

[30] Y. Yu and H. Liu, The factorable minimal surfaces, Proceedings of The Eleventh International Workshop on Diff. Geom., 11 (2007), 33-39.

Department of Mathematics, Firat University, 23119 Elazig, Turkey.

E-mail: meaydin@firat.edu.tr

Department of Mathematics, Namik Kemal University, 59000 Tekirdag.

E-mail: mergut@nku.edu.tr Turkey 